\title{
AN EXTENSION OF BITTNER AND URBAN'S THEOREM
}

\author{
YOUMING LIU AND JUNJIAN ZHAO
}

\begin{abstract}
A class of Besov spaces are characterized by the quadratic and cubic Hermite multiwavelets (K. Bittner and K. Urban, On interpolatory divergence-free wavelets, Math. Comp., 76 (2007), 903-929). That characterization has a limitation, because of the regularity restriction of the Hermite splines. In this paper, we extend Bittner and Urban's theorem by using Bspline wavelets with weak duals introduced in the paper: R. Q. Jia, J. Z. Wang and D. X. Zhou, Compactly supported wavelet bases for Sobolev spaces, Appl. Comput. Harmon. Anal., 15 (2003), 224-241.
\end{abstract}

\section{INTRODUCTION}

It is important in both theory and application to characterize function spaces by using wavelet bases. In particular, the characterization for Besov spaces interests mathematicians since many fundamental spaces (e.g., Sobolev, Hölder, Lipschitz spaces and etc.) are special examples of those spaces ([5]). In [1], Bittner and Urban study the following Besov spaces and the related vector Besov spaces:

Let $0<p, q \leq \infty$ and $s>0$,

$$
B B_{p, q}^{s}(\mathbb{R}):=\left\{f \in L_{p}(\mathbb{R}):|f|_{B B_{p, q}^{s}(\mathbb{R})}<\infty\right\}
$$

with the Besov (semi-)norm $\|f\|_{B B_{p, q}^{s}(\mathbb{R})}:=\|f\|_{L_{p}(\mathbb{R})}+|f|_{B B_{p, q}^{s}(\mathbb{R})}$. To define $|f|_{B B_{p, q}^{s}(\mathbb{R})}$, we need the classical difference operator $\Delta_{h}: \Delta_{h} f(\cdot):=f(\cdot+h)+f(\cdot)$, as well as $\Delta_{h}^{M} f:=\Delta_{h}\left(\Delta_{h}^{M-1} f\right)$ for a positive integer $M>1$. Moreover,

$$
\omega_{p}^{M}\left(f, 2^{-j}\right):=\sup _{|h| \leq 2^{-j}}\left\|\Delta_{h}^{M} f(\cdot)\right\|_{L_{p}(\mathbb{R})}
$$

denotes the $M$-th order smooth modulus of a function $f$. Then $|f|_{B B_{p, q}^{s}(\mathbb{R})}$ is defined as

$$
|f|_{B B_{p, q}^{s}(\mathbb{R})}:=\left\|\left(2^{j s} \omega_{p}^{M}\left(f, 2^{-j}\right)\right)_{j \in \mathbb{Z}}\right\|_{\ell_{q}},
$$

where $M \geq\lfloor s\rfloor+1$ and $\lfloor s\rfloor$ stands for the largest integer less than or equal to $s$.

Based on the quadratic or cubic Hermite multiwavelets, the authors of [1] characterize $B B_{p, q}^{s}$ by using the sequence norms

$$
\|a\|_{\ell_{p}} \quad \text { and } \quad\|b\|_{\ell_{p, q}^{s}}:=\left\|\left(2^{n\left(s+\frac{1}{2}-\frac{1}{p}\right)}\left\|\left(b_{n, k}\right)_{k \in \mathbb{Z}}\right\|_{\ell_{p}}\right)_{n \geq n_{0}}\right\|_{\ell_{q}}
$$

Received by the editor August 11, 2009 and, in revised form, July 18, 2011.

2010 Mathematics Subject Classification. Primary 42C40, 35Q30, 41A15.

Key words and phrases. Wavelet characterization, Besov spaces, completeness.

This work is supported by the National Natural Science Foundation of China (No. 10871012) and the Natural Science Foundation of Beijing (No. 1082003). 
for $a=\left(a_{k}\right)_{k \in \mathbb{Z}} \in \ell_{p}$ and $b=\left(b_{n, k}\right)_{n \geq n_{0}, k \in \mathbb{Z}} \in \ell_{p, q}^{s}$, where $\ell_{p, q}^{s}:=\left\{\left(c_{n, k}\right)_{n \geq n_{0}, k \in \mathbb{Z}}\right.$ : $\left.\left\|\left(2^{n\left(s+\frac{1}{2}-\frac{1}{p}\right)}\left\|\left(c_{n, k}\right)_{k \in \mathbb{Z}}\right\|_{\ell_{p}}\right)_{n \geq n_{0}}\right\|_{\ell_{q}}<+\infty\right\}$. However, due to the regularity restrictions of the Hermite splines, their characterization requires $\frac{1}{p}<s<\min \left\{3,1+\frac{1}{p}\right\}$ in the quadratic case and $1+\frac{1}{p}<s<\min \left\{4,2+\frac{1}{p}\right\}$ in the cubic case.

This current paper removes that restriction of $s$ by using B-spline wavelets with weak duals introduced in 9]. Because we establish some differential relations of those wavelets ([11]), divergence-free wavelets can be constructed. However, the result of this paper cannot be used to construct interpolating divergence-free wavelets, since it is shown in [1] that multi-wavelets are needed for that purpose.

Let $\mathbb{N}, \mathbb{Z}$ and $\mathbb{R}$ be the set of positive integers, the set of integers and the set of real numbers, respectively. Throughout this paper, we use $A \lesssim B$ to abbreviate that $A$ is bounded by a constant multiple of $B, A \gtrsim B$ is defined as $B \lesssim A$ and $A \sim B$ means $A \lesssim B$ and $B \lesssim A$. Let $L_{p}(\Omega)$ denote the classical Lebesgue spaces for a Lebesgue measurable set $\Omega \subseteq \mathbb{R}$ and $1 \leq p \leq \infty$. Write

$$
\langle f, g\rangle=\int_{\Omega} f(t) \overline{g(t)} d t
$$

for $f \in L_{p}(\Omega), g \in L_{p^{\prime}}(\Omega)$ with $\frac{1}{p}+\frac{1}{p^{\prime}}=1$. Similarly, $\ell_{p}:=\left\{a=\left(a_{k}\right)_{k \in \mathbb{Z}}\right.$ : $\left.\sum_{k}\left|a_{k}\right|^{p}<\infty\right\}$. For a Lebesgue measurable function $f$, the support of $f$ means the closure of $\{x \in \mathbb{R}: f(x) \neq 0\}$ (denoted by $\operatorname{Supp}(f)$ ), which is well defined up to a set of measure 0. As usual, $\phi_{r}$ stands for the B-spline scaling function of order $r$, i.e., $r$ convolutions of the characteristic function on the interval $[0,1]$,

$$
\phi_{r}(\cdot):=\underbrace{\chi_{[0,1]} * \chi_{[0,1]} * \cdots * \chi_{[0,1]}}_{r \text { functions }}(\cdot) .
$$

Here and after, we always define $f_{n, k}(\cdot):=2^{\frac{n}{2}} f\left(2^{n} \cdot-k\right)$ unless we specifically state otherwise. Let $\varphi:=\phi_{r}, \tilde{\varphi}:=\phi_{\theta}$ be B-spline functions with $r+\theta$ even and $V_{n}=$ $\operatorname{clos}_{L_{2}} \operatorname{span}\left\{\varphi_{n, k}\right\}_{k \in \mathbb{Z}}, \tilde{V}_{n}=\operatorname{clos}_{L_{2}} \operatorname{span}\left\{\tilde{\varphi}_{n, k}\right\}_{k \in \mathbb{Z}}$. Assume that the corresponding wavelet pair $\psi, \tilde{\psi}$ is given by

$$
\psi(x)=\sum_{k \in \mathbb{Z}}(-1)^{k} \overline{\mu(1-k)} \varphi(2 x-k), \quad \tilde{\psi}(x)=\sum_{k \in \mathbb{Z}}(-1)^{k} \overline{\nu(1-k)} \tilde{\varphi}(2 x-k)
$$

for $\mu(k)=\langle\tilde{\varphi}(x), \varphi(2 x-k)\rangle$ and $\nu(k)=\langle\varphi(x), \tilde{\varphi}(2 x-k)\rangle$. Then the reference 9] shows the following:

(1) $\langle\psi(\cdot), \tilde{\varphi}(\cdot-k)\rangle=\langle\varphi(\cdot), \tilde{\psi}(\cdot-k)\rangle=\left\langle\psi_{j, k}, \tilde{\psi}_{j^{\prime}, k^{\prime}}\right\rangle=0$ for $j \neq j^{\prime}$ and $k, k^{\prime} \in \mathbb{Z}$;

(2) $V_{n+1}=V_{n} \bigoplus W_{n}$ and $\tilde{V}_{n+1}=\tilde{V}_{n} \bigoplus \tilde{W}_{n}$. Here, $W_{n}=\operatorname{clos}_{L_{2}} \operatorname{span}\left\{\psi_{n, k}\right\}$, $\tilde{W}_{n}=\operatorname{clos}_{L_{2}} \operatorname{span}\left\{\tilde{\psi}_{n, k}\right\}$. Furthermore, $\left\{\psi_{n, k}\right\}_{n \geq n_{0}, k \in \mathbb{Z}}$ is a Riesz basis of $L_{2}(\mathbb{R})$;

(3) $\quad L_{2}(\mathbb{R})=V_{n} \oplus \tilde{V}_{n}^{\perp}$ and the projection operator $P_{n}$ (with range $V_{n}$ and null space $\tilde{V}_{n}^{\perp}$ ) satisfies $\left\|P_{n}\right\|_{L_{2}} \leq C$, independent of $n$.

From (3), we find easily that

$$
P_{n} f(x):=\sum_{k \in \mathbb{Z}} a_{n, k} \varphi_{n, k}(x),
$$

where $a_{n}=\left(a_{n, k}\right)_{k} \in \ell_{2}$ is the unique solution for $\left(a_{n} * \lambda\right)(k)=\left\langle f, \tilde{\varphi}_{n, k}\right\rangle$ with $\lambda(k)=\langle\varphi(\cdot), \tilde{\varphi}(\cdot-k)\rangle$. By $(2), Q_{n} f(x):=P_{n+1} f-P_{n} f$ can be written as

$$
Q_{n} f(x)=\sum_{k \in \mathbb{Z}} b_{n, k} \psi_{n, k}(x),
$$


where $b_{n}=\left(a_{n, k}\right)_{k} \in \ell_{2}$ is the unique solution for $\left(b_{n} * \tau\right)(k)=\left\langle Q_{n} f, \tilde{\psi}_{n, k}\right\rangle$ with $\tau(k)=\langle\psi(\cdot), \tilde{\psi}(\cdot-k)\rangle$.

Since both $\varphi$ and $\psi$ have compact support, we extend the above operators from $L_{2}(\mathbb{R})$ to $L_{p}(\mathbb{R})$ for $p \geq 1$ in section 3 . The purpose of this paper is to prove the following main result:

Theorem 1.1. Let $\varphi=\phi_{r}, \tilde{\varphi}=\phi_{\theta}$ with $r+\theta$ even and the corresponding wavelets $\psi, \tilde{\psi}$ be given in (1.1). Then for $1 \leq p \leq \infty, 0<q<\infty$ and $0<s<r-1+\frac{1}{p}$, each $f \in B B_{p, q}^{s}$ has the expansion

$$
f=\sum_{k \in \mathbb{Z}} a_{n_{0}, k} \varphi_{n_{0}, k}+\sum_{n=n_{0}}^{\infty} \sum_{k \in \mathbb{Z}} b_{n, k} \psi_{n, k}
$$

in $B B_{p, q}^{s}$, where $a_{n_{0}}$ and $b_{n}$ are the unique solution of $\left(a_{n_{0}} * \lambda\right)(k)=\left\langle f, \tilde{\varphi}_{n_{0}, k}\right\rangle$ and $\left(b_{n} * \tau\right)(k)=\left\langle f, \tilde{\psi}_{n, k}\right\rangle$, respectively. Moreover, there exist positive constants $C_{1}, C_{2}$ (depending on $r$ and $\theta$ ) such that

$$
\begin{aligned}
C_{1}\left(\left\|a_{n_{0}}\right\|_{\ell_{p}}+\|b\|_{\ell_{p, q}^{s}}\right) & \leq\left\|\sum_{k \in \mathbb{Z}} a_{n_{0}, k} \varphi_{n_{0}, k}+\sum_{n=n_{0}}^{\infty} \sum_{k \in \mathbb{Z}} b_{n, k} \psi_{n, k}\right\|_{B B_{p, q}^{s}} \\
& \leq C_{2}\left(\left\|a_{n_{0}}\right\|_{\ell_{p}}+\|b\|_{\ell_{p, q}^{s}}\right) .
\end{aligned}
$$

Since for each $s>0$, there always exists a positive integer $r$ such that $s<$ $r-1+\frac{1}{p}$, our characterization covers all finite $s>0$. Of course, our functions $\varphi$ and $\psi$ have large supports. In the next section, we conclude $\| \sum_{k \in \mathbb{Z}} a_{n_{0}, k} \varphi_{n_{0}, k}+$ $\sum_{n=n_{0}}^{\infty} \sum_{k \in \mathbb{Z}} b_{n, k} \psi_{n, k}\left\|_{B B_{p, q}^{s}} \lesssim\right\| a_{n_{0}}\left\|_{\ell_{p}}+\right\| b \|_{\ell_{p, q}^{s}}$, which is an easy exercise in the characterization of function spaces. Section 3 is devoted to showing $\left\|a_{n_{0}}\right\|_{\ell_{p}}+$ $\|b\|_{\ell_{p, q}^{s}} \lesssim\|f\|_{B B_{p, q}^{s}}$ for $f \in B B_{p, q}^{s},\left(a_{n_{0}} * \lambda\right)(k)=\left\langle f, \tilde{\varphi}_{n_{0}, k}\right\rangle$ and $\left(b_{n} * \tau\right)(k)=$ $\left\langle f, \tilde{\psi}_{n, k}\right\rangle$. In the last part, we prove that (1.3) holds in $B B_{p, q}^{s}$ for each $f \in B B_{p, q}^{s}$.

\section{UPPER BOUND}

The estimation for the upper bound is usually much easier than that of the lower bound. In this section, we establish the upper bound in (1.4), for which we need the following simple lemma.

Lemma 2.1. If $1 \leq p \leq \infty, 0<q \leq \infty$, then the B-spline $\phi_{r} \in B B_{p, q}^{\sigma}$ for $\sigma<r-1+\frac{1}{p}$.

Proof. Since $r$ is a positive integer and $\sigma<r,\lfloor\sigma\rfloor+1 \leq r$ and one can take $M=r$, in order to show $\phi_{r} \in B B_{p, q}^{\sigma}$, i.e., $\left|\phi_{r}\right|_{B B_{p, q}^{\sigma}}=\left\|\left(2^{j \sigma} \omega_{p}^{r}\left(\phi_{r}, 2^{-j}\right)\right)_{j \in \mathbb{Z}}\right\|_{\ell_{q}}<\infty$. Clearly, $\omega_{p}^{r}\left(\phi_{r}, 2^{-j}\right) \leq C\left\|\phi_{r}\right\|_{p}$ and $\left\|\left(2^{j \sigma} \omega_{p}^{r}\left(\phi_{r}, 2^{-j}\right)\right)_{j \leq 0}\right\|_{\ell_{q}} \lesssim\left\|\phi_{r}\right\|_{p}$. Then it is sufficient to show that

$$
\left\|\left(2^{j \sigma} \omega_{p}^{r}\left(\phi_{r}, 2^{-j}\right)\right)_{j \geq 0}\right\|_{\ell_{q}}<\infty .
$$

Since $\Delta_{h}^{r} f(\cdot):=\Delta\left(\Delta_{h}^{r-1} f\right)(\cdot)=\int_{0}^{1} h\left(\Delta_{h}^{r-1} f\right)^{\prime}(\cdot+s h) d s=h \int_{0}^{1} \Delta_{h}^{r-1} f^{\prime}(\cdot+s h) d s$, one knows that

$$
\left\|\Delta_{h}^{r} f\right\|_{p} \leq|h| \int_{0}^{1}\left\|\Delta_{h}^{r-1} f^{\prime}(\cdot+s h)\right\|_{p} d s=|h| \int_{0}^{1}\left\|\Delta_{h}^{r-1} f^{\prime}(\cdot)\right\|_{p} d s
$$

by the integral Minkowski inequality. Moreover, the definition of $\omega_{p}^{r}(f, t)$ implies that

$$
\omega_{p}^{r}\left(\phi_{r}, 2^{-j}\right) \leq 2^{-j} \omega_{p}^{r-1}\left(\phi_{r}^{\prime}, 2^{-j}\right) \leq 2^{-j(r-1)} \omega_{p}^{1}\left(\phi_{r}^{(r-1)}, 2^{-j}\right) .
$$


On the other hand, $\left\|\phi_{1}(\cdot+h)-\phi_{1}(\cdot)\right\|_{p} \lesssim|h|^{\frac{1}{p}}$ shows $\omega_{p}^{1}\left(\phi_{1}, 2^{-j}\right) \lesssim 2^{-\frac{j}{p}}$. Note that $\phi_{r}^{\prime}(\cdot)=\phi_{r-1}(\cdot)-\phi_{r-1}(\cdot-1)$ and $\phi_{r}^{(r-1)}$ is a linear combination of $\phi_{1}(\cdot-k)$. Then, $\omega_{p}^{r}\left(\phi_{r}, 2^{-j}\right) \lesssim 2^{-j\left(r-1+\frac{1}{p}\right)}$ and $2^{j \sigma} \omega_{p}^{r}\left(\phi_{r}, 2^{-j}\right) \lesssim 2^{-j\left(r-1+\frac{1}{p}-\sigma\right)}$. This with the assumption $\sigma<r-1+\frac{1}{p}$ leads to (2.1), and the proof is completed.

To establish the upper bounds, we need the following result:

Lemma 2.2 ([1, Lemma 3.4]). Let $0<p, q \leq \infty$ and $\phi \in B B_{p, q}^{\sigma}$ be compactly supported. Then for $0<s<\sigma$,

$$
\begin{gathered}
\left\|\sum_{k \in \mathbb{Z}} a_{n, k} \phi\left(2^{n} \cdot-k\right)\right\|_{B B_{p, q}^{s}} \lesssim 2^{n\left(s-\frac{1}{p}\right)}\left\|\left(a_{n, k}\right)_{k}\right\|_{\ell_{p}}, \\
\left\|\sum_{n \geq n_{0}} \sum_{k \in \mathbb{Z}} a_{n, k} \phi\left(2^{n} \cdot-k\right)\right\|_{B B_{p, q}^{s}} \lesssim\left\|\left(2^{n\left(s-\frac{1}{p}\right)}\left\|\left(a_{n, k}\right)_{k \in \mathbb{Z}}\right\|_{\ell_{p}}\right)_{n \geq n_{0}}\right\|_{\ell_{q}} .
\end{gathered}
$$

Note that $\psi(\cdot)$ is a linear combination of $\varphi(\cdot-k)$. Then the following result follows from Lemma 2.1 and Lemma 2.2.

Theorem 2.1. Let $\varphi=\phi_{r}$ and $\psi$ be the wavelet given in Section 1 . If $1 \leq p \leq \infty$, $0<q \leq \infty, 0<s<r-1+\frac{1}{p}$. Then, for $a_{n_{0}} \in \ell_{p}$ and $b \in \ell_{p, q}^{s}$,

$$
\left\|\sum_{k \in \mathbb{Z}} a_{n_{0}, k} \varphi_{n, k}(\cdot)+\sum_{n \geq n_{0}} \sum_{k \in \mathbb{Z}} b_{n, k} \psi_{n, k}(\cdot)\right\|_{B B_{p, q}^{s}} \lesssim\left\|a_{n_{0}}\right\|_{\ell_{p}}+\|b\|_{\ell_{p, q}^{s}} .
$$

\section{LOWER BOUND}

This part is devoted to the establishment of the lower bound in (1.4). As in [2, we shall end it by establishing the relation between projection operators and the modulus of smoothness. The following lemma is frequently used. We include a short proof for completeness, although similar versions appear in some other references (with slightly different assumptions).

Lemma 3.1. Let $p \geq 1, \frac{1}{p}+\frac{1}{p^{\prime}}=1$ and $\varphi \in L_{p^{\prime}}(\mathbb{R}) \cap L_{p}(\mathbb{R})$ with compact support. If $f \in L_{p}(\mathbb{R})$ and $c=\left(c_{k}\right) \in \ell_{p}$, then

$$
\left\|\left(\left\langle f, \varphi_{n, k}\right\rangle\right)\right\|_{\ell_{p}} \lesssim 2^{n\left(\frac{1}{2}-\frac{1}{p^{\prime}}\right)}\|f\|_{p}, \quad \text { and } \quad\left\|\sum_{k} c_{k} \varphi_{n, k}\right\|_{p} \lesssim 2^{n\left(\frac{1}{2}-\frac{1}{p}\right)}\|c\|_{\ell_{p}} .
$$

In particular, when $\varphi=\phi_{r}$,

$$
\left\|\sum_{k} c_{k} \varphi_{n, k}\right\|_{p} \sim 2^{n\left(\frac{1}{2}-\frac{1}{p}\right)}\|c\|_{\ell_{p}}
$$

Proof. One proves the lemma for $p \in(1, \infty)$; similar arguments lead to the cases for $p=1, \infty$. Assume Supp $\varphi \subseteq[a, b]$, then

$$
|\langle f(\cdot), \varphi(\cdot-k)\rangle|^{p} \leq \int_{a}^{b}|f(x+k)|^{p} d x\left(\int_{a}^{b}|\varphi(x)|^{p^{\prime}} d x\right)^{\frac{p}{p^{\prime}}} \lesssim \int_{a+k}^{b+k}|f(x)|^{p} d x
$$

and $\|(\langle f(\cdot), \varphi(\cdot-k)\rangle)\|_{\ell_{p}} \lesssim\|f\|_{p}$. In general,

$$
\begin{aligned}
\left\|\left(\left\langle f(\cdot), \varphi_{n, k}(\cdot)\right\rangle\right)_{k}\right\|_{\ell_{p}} & =2^{-\frac{n}{2}}\left\|\left(\left\langle f\left(2^{-n} \cdot\right), \varphi(\cdot-k)\right\rangle\right)_{k}\right\|_{\ell_{p}} \lesssim 2^{-\frac{n}{2}}\left\|f\left(2^{-n} \cdot\right)\right\|_{p} \\
& =2^{-\frac{n}{2}} 2^{\frac{n}{p}}\|f\|_{p}=2^{n\left(\frac{1}{2}-\frac{1}{p^{\prime}}\right)}\|f\|_{p},
\end{aligned}
$$


which is the first inequality in (3.1). Note that

$$
\left\|\sum_{k} c_{k} \varphi_{n, k}(x)\right\|_{p}^{p}=2^{\frac{n p}{2}-n}\left\|\sum_{k} c_{k} \varphi(x-k)\right\|_{p}^{p}
$$

and

$$
\begin{aligned}
\left\|\sum_{k} c_{k} \varphi(x-k)\right\|_{p}^{p} & =\sum_{k^{\prime}} \int_{k^{\prime}}^{k^{\prime}+1}\left|\sum_{k=k^{\prime}+1-\lceil b\rceil}^{k^{\prime}-\lfloor a\rfloor} c_{k} \varphi(x-k)\right|^{p} d x \\
& \lesssim \sum_{k^{\prime}} \int_{k^{\prime}}^{k^{\prime}+1} \sum_{k=k^{\prime}+1-\lceil b\rceil}^{k^{\prime}-\lfloor a\rfloor}\left|c_{k}\right|^{p}|\varphi(x-k)|^{p} d x \lesssim\|c\|_{\ell_{p}}^{p} .
\end{aligned}
$$

Then $\left\|\sum_{k} c_{k} \varphi_{n, k}\right\|_{p} \lesssim 2^{n\left(\frac{1}{2}-\frac{1}{p}\right)}\|c\|_{\ell_{p}}$, which is the second part of (3.1). Here, $\lfloor s\rfloor$ $(\lceil s\rceil)$ denote the largest (smallest) integer less (greater) than or equal to $s$.

In particular, for $\varphi=\phi_{r}$, because $\left\{\phi_{r}(x-k): k^{\prime}-r+1 \leq k \leq k^{\prime}\right\}$ is linearly independent on $\left[k^{\prime}, k^{\prime}+1\right]$, the classical functional analysis tells us (two norms are always equivalent on $r$-dimensional linear spaces)

$$
\left\|\sum_{k} c_{k} \phi_{r}(x-k)\right\|_{L_{p}\left(\left[k^{\prime}, k^{\prime}+1\right]\right)} \sim\left\|\left(c_{k}\right)_{k=k^{\prime}-r+1}^{k^{\prime}}\right\|_{\ell_{p}},
$$

where the constant depends only on $r$. Moreover,

$$
\begin{aligned}
\left\|\sum_{k} c_{k} \varphi(x-k)\right\|_{L_{p}}^{p} & =\sum_{k^{\prime} \in \mathbb{Z}}\left\|\sum_{k} c_{k} \varphi(x-k)\right\|_{L_{p}\left(\left[k^{\prime}, k^{\prime}+1\right]\right)}^{p} \\
& \sim \sum_{k^{\prime} \in \mathbb{Z}}\left\|\left(c_{k}\right)_{k=k^{\prime}-r+1}^{k^{\prime}}\right\|_{\ell_{p}}^{p} \sim\|c\|_{\ell_{p}}^{p} .
\end{aligned}
$$

Finally,

$$
\left\|\sum_{k} c_{k} \varphi_{n, k}(x)\right\|_{p}^{p}=2^{n\left(\frac{p}{2}-1\right)}\left\|\sum_{k} c_{k} \varphi(x-k)\right\|_{p}^{p} \sim 2^{n\left(\frac{p}{2}-1\right)}\|c\|_{\ell_{p}}^{p}
$$

and (3.2) results.

To extend the projection operators $P_{n}$ and $Q_{n}$ (introduced in the first section) from $L_{2}(\mathbb{R})$ to $L_{p}(\mathbb{R})$ for $p \geq 1$, we use the following lemma:

Lemma 3.2. Let $\varphi=\phi_{r}, \tilde{\varphi}=\phi_{\theta}$ with $r+\theta$ even, let $\psi$ and $\tilde{\psi}$ be the corresponding wavelets given in (1.1). Define $V_{n}:=\left\{\sum_{k} a_{k} \varphi_{n, k}:\left(a_{k}\right) \in \ell_{p}\right\}$ and $W_{n}:=\left\{\sum_{k} b_{k} \psi_{n, k}:\left(b_{k}\right) \in \ell_{p}\right\}$ in the sense of $L_{p}(\mathbb{R})$. Then

$$
V_{n} \bigoplus W_{n}=V_{n+1},
$$

where " $\bigoplus$ " denotes the direct sum.

Proof. By Lemma 3.1, both $V_{n}$ and $W_{n}$ are well-defined subspaces of $L_{p}(\mathbb{R})$. Note that $f \in V_{0}$ or $W_{0}$ is equivalent to $f\left(2^{n} \cdot\right) \in V_{n}$ or $W_{n}$. Then it is sufficient to show

$$
V_{0} \bigoplus W_{0}=V_{1} \text {. }
$$

Since $\varphi$ has compact support and $\varphi(x)=\sum_{l=0}^{r} h_{l} \varphi(2 x-l)$, one has $\sum_{k} a_{k} \varphi(x-k)=$ $\sum_{l=0}^{r} h_{l} \sum_{k} a_{k} \varphi(2 x-2 k-l) \in V_{1}$ and $V_{0} \subseteq V_{1}$. Similarly, $W_{0} \subseteq W_{1}$ and therefore $V_{0}+W_{0} \subseteq V_{1}$.

Next, one shows $V_{1} \subseteq V_{0}+W_{0}$ : 
According to the proof of Lemma 4.3 in [9], there exist $\left(c_{l}\right)_{l \in \mathbb{Z}},\left(d_{l}\right)_{l \in \mathbb{Z}},\left(c_{l}^{\prime}\right)_{l \in \mathbb{Z}}$, $\left(d_{l}^{\prime}\right)_{l \in \mathbb{Z}} \in \ell_{2}(\mathbb{Z})$ such that

$$
\varphi(2 x)=\sum_{l} c_{l} \varphi(x-l)+\sum_{l} d_{l} \psi(x-l)
$$

and

$$
\varphi(2 x-1)=\sum_{l} c_{l}^{\prime} \varphi(x-l)+\sum_{l} d_{l}^{\prime} \psi(x-l) .
$$

By combining (3.4) with $\langle\varphi(x), \tilde{\psi}(x-l)\rangle=\langle\psi(x), \tilde{\varphi}(x-l)\rangle=0$, one has

$$
\left\langle\varphi(2 x), \tilde{\varphi}\left(x-l^{\prime}\right)\right\rangle=(c * \lambda)\left(l^{\prime}\right), \quad\left\langle\varphi(2 x), \tilde{\psi}\left(x-l^{\prime}\right)\right\rangle=(d * \tau)\left(l^{\prime}\right),
$$

where $\lambda(k):=\langle\varphi(\cdot), \tilde{\varphi}(\cdot-k)\rangle$ and $\tau(k):=\langle\psi(\cdot), \tilde{\psi}(\cdot-k)\rangle$. Because $\hat{\lambda}(\xi):=$ $\sum_{k} \lambda(k) e^{i k \xi} \neq 0$ for each $\xi \in \mathbb{R}([9]$, Lemma 2.2$)$ and $\lambda(k)=0$ except for finitely many $k$ due to the compactness of $\varphi$ and $\tilde{\varphi}$, one gets

$$
\hat{\eta}(\xi):=\frac{1}{\hat{\lambda}(\xi)}=\sum_{k} \eta(k) e^{i k \xi}
$$

with $(\eta(k))_{k} \in \ell_{1}$, thanks to Wiener's Lemma (see [10]). Note that both $\varphi$ and $\tilde{\varphi}$ have compact supports. Then $\left(\left\langle\varphi(2 x), \tilde{\varphi}\left(x-l^{\prime}\right)\right\rangle\right)_{l^{\prime}} \in \ell_{1}$. Moreover, $c=\eta *$ $\left(\left\langle\varphi(2 x), \tilde{\varphi}\left(x-l^{\prime}\right)\right\rangle\right) \in \ell_{1}$. Similarly, since $\hat{\tau}(\xi) \neq 0([9]$, Lemma 4.2), one sees that $d \in \ell_{1}$. Hence, $c, d \in \ell_{p}$ for $p \geq 1$ and $\varphi(2 x) \in V_{0}+W_{0}$. The same arguments (with (3.5)) lead to $\varphi(2 x-1) \in V_{0}+W_{0}$.

In general, let $f \in V_{1}$ and $f(x)=\sum_{k} a_{k} \varphi(2 x-2 k)+\sum_{k} b_{k} \varphi(2 x-2 k-1)$ with $\left(a_{k}\right) \in \ell_{p},\left(b_{k}\right) \in \ell_{p}$. Then, by $(3.4)$,

$$
\begin{aligned}
\sum_{k} a_{k} \varphi(2 x-2 k) & =\sum_{k} a_{k} \sum_{l} c_{l} \varphi(x-k-l)+\sum_{k} a_{k} \sum_{l} d_{l} \psi(x-k-l) \\
& =\sum_{l}(a * c)(l) \varphi(x-l)+\sum_{l}(a * d)(l) \psi(x-l) .
\end{aligned}
$$

Since $c, d \in \ell_{1}$ and $a \in \ell_{p},(a * c) \in \ell_{p},(a * d) \in \ell_{p}$ and $\sum_{k} a_{k} \varphi(2 x-2 k) \in V_{0}+W_{0}$. Analogously, by (3.5), $\sum_{k} b_{k} \varphi(2 x-2 k-1) \in V_{0}+W_{0}$. Hence, $f \in V_{0}+W_{0}$, which implies $V_{1} \subseteq V_{0}+W_{0}$.

To get (3.3), it remains to conclude $a_{k}=b_{k}=0$, when $\left(a_{k}\right),\left(b_{k}\right) \in \ell_{p}$ and

$$
\sum_{k} a_{k} \varphi(x-k)+\sum_{k} b_{k} \psi(x-k)=0 .
$$

Clearly, $(a * \lambda)(l)=(b * \tau)(l)=0$ for each $l \in \mathbb{Z}$, and furthermore, $\hat{a}(\xi) \hat{\lambda}(\xi)=$ $\hat{b}(\xi) \hat{\tau}(\xi)=0$. Note that since $\hat{\lambda}(\xi) \neq 0, \hat{\tau}(\xi) \neq 0$ for each $\xi \in \mathbb{R}$, one has $\hat{a}(\xi)=$ $\hat{b}(\xi)=0$ and $a=b=0$. This completes the proof of (3.3) and finally Lemma 3.2 follows.

Let $f \in L_{p}(\mathbb{R})$. Then $c_{n}:=\left(\left\langle f, \tilde{\varphi}_{n, k}\right\rangle\right)_{k} \in \ell_{p}$ due to Lemma 3.1. From the proof of Lemma 3.2, $\left(a_{n} * \lambda\right)(k)=c_{n}(k)$ has the unique solution $a_{n} \in \ell_{p}$. Now, we define

$$
P_{n} f(x)=\sum_{k \in \mathbb{Z}} a_{n, k} \varphi_{n, k}(x) \in V_{n},
$$

which is formally the same as in (1.2). Moreover, the proof of Lemma 3.2 shows

$$
\frac{1}{\|\lambda\|_{\ell_{1}}}\left\|c_{n}\right\|_{\ell_{p}} \leq\left\|a_{n}\right\|_{\ell_{p}} \leq\|\eta\|_{\ell_{1}}\left\|c_{n}\right\|_{\ell_{p}}
$$


Define $Q_{n} f:=P_{n+1} f-P_{n} f$. Then there exists a unique $\left(b_{n, k}\right)_{k} \in \ell_{p}$ such that

$$
Q_{n} f=\sum_{k} b_{n, k} \psi_{n, k} \in W_{n}
$$

according to Lemma 3.2. Here, $d_{n}(k):=\left\langle Q_{n} f, \tilde{\psi}_{n, k}\right\rangle$ satisfies $\left(b_{n} * \tau\right)(k)=d_{n}(k)$ and

$$
\frac{1}{\|\tau\|_{\ell_{1}}}\left\|d_{n}\right\|_{\ell_{p}} \leq\left\|b_{n}\right\|_{\ell_{p}} \leq\|\sigma\|_{\ell_{1}}\left\|d_{n}\right\|_{\ell_{p}}
$$

with $\hat{\sigma}(\xi):=\frac{1}{\hat{\tau}(\xi)}$. In order to prove Lemma 3.4, we need the next lemma, which can be found in [7] (Theorem 3.2) and [8]:

Lemma 3.3. Let $\varphi=\phi_{r}$. Then there exist a continuous, compactly supported function sequence $\left\{\gamma_{n, k}\right\}_{n \geq n_{0}, k \in \mathbb{Z}}$ (not necessarily generated by one function) such that for $f \in L_{p}, D_{n} f(x)=\sum_{k \in \mathbb{Z}}\left\langle f, \gamma_{n, k}\right\rangle \varphi_{n, k}(x)$ satisfies

$$
\left\|f-D_{n} f\right\|_{p} \lesssim \omega_{p}^{r}\left(f, 2^{-n}\right) .
$$

Now, we show some properties of $P_{n}$, which will be used in the proof of Theorem 3.1 and Theorem 4.1.

Lemma 3.4. If $f \in L_{p}(\mathbb{R})$, then

(i) $\quad P_{n} P_{l} f=P_{l} P_{n} f=P_{l} f$, when $l \leq n$;

(ii) $\quad\left\|P_{n} f\right\|_{p} \leq C\|f\|_{p}$ for some $C$ (independent of $f$ and $n$ );

(iii) $\left\|f-P_{n} f\right\|_{p} \lesssim \omega_{p}^{r}\left(f, 2^{-n}\right)$.

Proof. (i) It is easy to see $P_{n} v_{n}=v_{n}$ for $v_{n} \in V_{n}$; in fact, assume $v_{n}=\sum_{k} a_{n, k}^{\prime} \varphi_{n, k}$ and $P_{n} v_{n}=\sum_{k} a_{n, k} \varphi_{n, k}$ with $\left(a_{n, k}^{\prime}\right)_{k},\left(a_{n, k}\right)_{k} \in \ell_{p}$, then

$$
\left(a_{n} * \lambda\right)(l)=\left\langle v_{n}, \tilde{\varphi}_{n, l}\right\rangle=\left\langle\sum_{k} a_{n, k}^{\prime} \varphi_{n, k}, \tilde{\varphi}_{n, l}\right\rangle=\left(a_{n}^{\prime} * \lambda\right)(l)
$$

by the definition of $P_{n}$. Furthermore, $\hat{a}_{n}^{\prime}(\xi) \lambda(\xi)=\hat{a}_{n}(\xi) \lambda(\xi)$, which implies $a_{n}=a_{n}^{\prime}$ by $\hat{\lambda}(\xi) \neq 0$ for each $\xi \in \mathbb{R}$. This shows $P_{n} v_{n}=v_{n}$ for $v_{n} \in V_{n}$. Then $P_{n} P_{l} f=P_{l} f$, when $l \leq n$. To show $P_{l} P_{n} f=P_{l} f$, one observes

$$
P_{n} f=P_{l} f+\sum_{j=l}^{n-1} Q_{j} f
$$

from the definition of $Q_{n}$. On the other hand, it is easy to see that $P_{l} g=0$ for $g \in W_{j}$ with $j \geq l$, since $c_{l}(k)=\left\langle g, \tilde{\varphi}_{l, k}\right\rangle=0$. Hence,

$$
P_{l} P_{n} f=P_{l} P_{l} f+\sum_{j=l}^{n-1} P_{l}\left(Q_{j} f\right)=P_{l} f+\sum_{j=l}^{n-1} P_{l}\left(Q_{j} f\right)=P_{l} f .
$$

(ii) By combining (3.6) with (3.1), one has

$$
\left\|P_{n} f\right\|_{p} \lesssim 2^{n\left(\frac{1}{2}-\frac{1}{p}\right)}\left\|a_{n}\right\|_{\ell_{p}} \leq C 2^{n\left(\frac{1}{2}-\frac{1}{p}\right)}\left\|c_{n}\right\|_{\ell_{p}} .
$$

Clearly, Lemma 3.1 shows that $\left\|c_{n}\right\|_{\ell_{p}}:=\left\|\left(\left\langle f, \tilde{\varphi}_{n, k}\right\rangle\right)_{k}\right\|_{\ell_{p}} \lesssim 2^{n\left(\frac{1}{2}-\frac{1}{p^{\prime}}\right)}\|f\|_{p}$ with $\frac{1}{p}+\frac{1}{p^{\prime}}=1$. Then, $\left\|P_{n} f\right\|_{p} \leq C\|f\|_{p}$. 
(iii) Since $D_{n} f \in V_{n}, P_{n} D_{n} f=D_{n} f$ and $\left\|D_{n} f-P_{n} f\right\|_{p}=\left\|P_{n} D_{n} f-P_{n} f\right\|_{p} \leq$ $C\left\|f-D_{n} f\right\|_{p}$ due to (ii). Moreover,

$$
\left\|f-P_{n} f\right\|_{p} \leq\left\|f-D_{n} f\right\|_{p}+\left\|D_{n} f-P_{n} f\right\|_{p} \lesssim\left\|f-D_{n} f\right\|_{p} \lesssim \omega_{p}^{r}\left(f, 2^{-n}\right),
$$

where the last inequality comes from Lemma 3.3.

Now, we are ready to give the lower bounds:

Theorem 3.1. Let $f \in B B_{p, q}^{s}$ for $s>0$ with $1 \leq p \leq \infty, 0<q \leq \infty,\left(a_{n_{0}} * \lambda\right)(k)=$ $\left\langle f, \tilde{\varphi}_{n_{0}, k}\right\rangle$ and $\left(b_{n} * \tau\right)(k)=\left\langle Q_{n} f, \tilde{\psi}_{n, k}\right\rangle$ for $n \geq n_{0}$. Then

$$
\left\|a_{n_{0}}\right\|_{\ell_{p}}+\|b\|_{\ell_{p, q}^{s}} \lesssim\|f\|_{B B_{p, q}^{s}} .
$$

Proof. By (3.6) and Lemma 3.1, one knows that $\left\|a_{n_{0}}\right\|_{\ell_{p}} \lesssim\left\|\left(\left\langle f, \tilde{\varphi}_{n_{0}, k}\right\rangle\right)\right\|_{\ell_{p}}$ $\leq 2^{n_{0}\left(\frac{1}{2}-\frac{1}{p^{\prime}}\right)}\|f\|_{p}$ and therefore $\left\|a_{n_{0}}\right\|_{\ell_{p}} \lesssim\|f\|_{B B_{p, q}^{s}}$. In order to show $\|b\|_{\ell_{p, q}^{s}} \lesssim$ $\|f\|_{B B_{p, q}^{s}}$, it is sufficient to conclude $\|d\|_{\ell_{p, q}^{s}} \lesssim\|f\|_{B B_{p, q}^{s}}$, due to (3.7). According to (3.1), one gets

$$
\left\|\left(d_{n, k}\right)_{k}\right\|_{\ell_{p}}:=\left\|\left(\left\langle Q_{n} f(x), \tilde{\psi}_{n, k}(\cdot)\right\rangle\right)_{k}\right\|_{\ell_{p}} \lesssim\left\|Q_{n} f(x)\right\|_{L_{p}} 2^{n\left(\frac{1}{2}-\frac{1}{p^{\prime}}\right)} .
$$

Using (iii) in Lemma 3.4, one has

$$
\begin{aligned}
\left\|Q_{n} f\right\|_{p}:= & \left\|P_{n+1} f(x)-P_{n} f(x)\right\|_{p} \leq\left\|f-P_{n+1} f\right\|_{p} \\
& +\left\|f-P_{n} f\right\|_{p} \lesssim \omega_{p}^{r}\left(f, 2^{-n}\right) .
\end{aligned}
$$

Moreover, (3.8) and (3.9) imply that $\left\|\left(d_{n, k}\right)_{k}\right\|_{\ell_{p}} \lesssim 2^{n\left(\frac{1}{2}-\frac{1}{p^{\prime}}\right)} \omega_{p}^{r}\left(f, 2^{-n}\right)$. Finally, $\|d\|_{\ell_{p, q}^{s}}:=\left\|\left(2^{n\left(s+\frac{1}{2}-\frac{1}{p}\right)}\left\|\left(d_{n, k}\right)_{k}\right\|_{\ell_{p}}\right)_{n \geq n_{0}}\right\|_{\ell_{q}} \lesssim\left\|\left(2^{n s} \omega_{p}^{r}\left(f, 2^{-n}\right)\right)_{n \geq n_{0}}\right\|_{\ell_{q}} \leq\|f\|_{B B_{p, q}^{s}}$. This completes the proof.

\section{Completeness}

In this section, we prove the completeness of $\left\{\varphi_{n_{0}, k}, \psi_{n, k}, k \in \mathbb{Z}, n \geq n_{0}\right\}$ in $B B_{p, q}^{s}$. First, we give a simple property for $P_{l} f$. A similar result can be found in [2] in which $P_{l} f$ is defined by biorthogonal scaling functions $\varphi$ and $\tilde{\varphi}$ with $\langle\varphi(\cdot), \tilde{\varphi}(\cdot-$ $k)\rangle=\delta_{k, 0}$.

Lemma 4.1. For $1 \leq p \leq \infty$ and $f \in L_{p}(\mathbb{R}), \omega_{p}^{r}\left(P_{l} f, 2^{-n}\right) \lesssim 2^{(l-n)\left(r-1+\frac{1}{p}\right)}\left\|P_{l} f\right\|_{p}$ for $l \leq n$.

Proof. It is sufficient to show Lemma 4.1 only for $1 \leq p<\infty$, the case for $p=\infty$ is similar. By $P_{l} f(x)=\sum_{k \in \mathbb{Z}} a_{l, k} \varphi_{l, k}(x)$, one knows that

$$
\left\|\Delta_{h}^{r} P_{l} f\right\|_{p}^{p}=\left\|\sum_{k \in \mathbb{Z}} a_{l, k} \Delta_{h}^{r} \varphi_{l, k}(x)\right\|_{p}^{p}=2^{l\left(\frac{p}{2}-1\right)} \int\left|\sum_{k \in \mathbb{Z}} a_{l, k} \Delta_{2^{l} h}^{r} \varphi(x-k)\right|^{p} d x .
$$

Because $\varphi=\phi_{r}$ has compact support, there exist fixed $L_{1}<L_{2}$ such that

$$
\begin{aligned}
\int\left|\sum_{k \in \mathbb{Z}} a_{l, k} \Delta_{2^{l} h}^{r} \varphi(x-k)\right|^{p} d x & =\sum_{k^{\prime} \in \mathbb{Z}} \int_{k^{\prime}}^{k^{\prime}+1}\left|\sum_{k=k^{\prime}+L_{1}}^{k^{\prime}+L_{2}} a_{l, k} \Delta_{2^{l} h}^{r} \varphi(x-k)\right|^{p} d x \\
& \lesssim \sum_{k^{\prime} \in \mathbb{Z}} \int_{k^{\prime}}^{k^{\prime}+1} \sum_{k=k^{\prime}+L_{1}}^{k^{\prime}+L_{2}}\left|a_{l, k}\right|^{p}\left|\Delta_{2^{l} h}^{r} \varphi(x-k)\right|^{p} d x
\end{aligned}
$$


for $\left|2^{l} h\right| \leq 1$. Note that $2^{l} 2^{-n} \leq 1$ for $l \leq n$, and the proof of Lemma 2.1 implies $\left\|\Delta_{h}^{r} \varphi\right\|_{p} \lesssim|h|^{r-1+\frac{1}{p}}$ for $\varphi=\phi_{r}$. Then $\left\|\Delta_{h}^{r} P_{l} f\right\|_{p} \lesssim 2^{l\left(\frac{1}{2}-\frac{1}{p}\right)}\left|2^{l} h\right|^{r-1+\frac{1}{p}}\left\|\left(a_{l, k}\right)_{k}\right\|_{\ell_{p}}$. Now, it follows that

$$
\omega_{p}^{r}\left(P_{l} f, 2^{-n}\right) \lesssim 2^{l\left(\frac{1}{2}-\frac{1}{p}\right)} 2^{(l-n)\left(r-1+\frac{1}{p}\right)}\left\|\left(a_{l, k}\right) k\right\|_{\ell_{p}} .
$$

On the other hand, (3.2) tells us that $\left\|P_{l} f\right\|_{p} \sim 2^{l\left(\frac{1}{2}-\frac{1}{p}\right)}\left\|\left(a_{l, k}\right)_{k}\right\|_{\ell_{p}}$. Hence, $\omega_{p}^{r}\left(P_{l} f, 2^{-n}\right) \lesssim 2^{(l-n)\left(r-1+\frac{1}{p}\right)}\left\|P_{l} f\right\|_{p}$ holds.

To prove Theorem 4.1, we need the discrete Hardy inequality:

Lemma 4.2 ([4, Lemma 4.2]). Given two positive sequences $a=\left(a_{j}\right)_{j \in \mathbb{Z}}$ and $b=\left(b_{j}\right)_{j \in \mathbb{Z}}$, if there exist $C_{0}>0, \mu>0$ such that $b_{j} \leq C_{0}\left(\sum_{r=j}^{\infty} a_{r}^{\mu}\right)^{\frac{1}{\mu}}$, Then $\|b\|_{\ell_{q}^{s}} \lesssim\|a\|_{\ell_{q}^{s}}$ holds for each $s>0$ and $0<q \leq+\infty$. Here $\ell_{q}^{s}:=\left\{\left(a_{j}\right)_{j}:\left(2^{j s} a_{j}\right)_{j} \in\right.$ $\left.\ell_{q}\right\}$ and $\left\|\left(a_{j}\right)_{j}\right\|_{\ell_{q}^{s}}=\left\|\left(2^{j s} a_{j}\right)_{j}\right\|_{\ell_{q}}$.

Theorem 4.1. Let $f \in B B_{p, q}^{s}$ with $1 \leq p \leq \infty, 0<q<\infty$ and $s \in\left(0, r-1+\frac{1}{p}\right)$. Then $f=P_{n_{0}} f+\sum_{n \geq n_{0}} Q_{n} f$ holds in $B B_{p, q}^{s}$.

Proof. Motivated by [3, one introduces

$$
D B_{p, q}^{s}(\mathbb{R}):=\left\{f \in L_{p}(\mathbb{R}):\|f\|_{D B_{p, q}^{s}(\mathbb{R})}:=\left\|P_{n_{0}} f\right\|_{L_{p}(\mathbb{R})}+|f|_{D B_{p, q}^{s}}<\infty\right\},
$$

where $|f|_{D B_{p, q}^{s}}=\left\|\left(2^{n s}\left\|f-P_{n} f\right\|_{L_{p}(\mathbb{R})}\right)_{n \geq n_{0}}\right\|_{\ell_{q}}$. By Lemma 3.4 (i), $f \in D B_{p, q}^{s}$ implies $P_{l} f \in D B_{p, q}^{s}$ for $l \geq n_{0}$. Similarly, $P_{n_{0}}\left(P_{l} f-f\right)=0$ and

$$
\begin{aligned}
\left|P_{l} f-f\right|_{D B_{p, q}^{s}} & =\left\|\left(2^{n s}\left\|\left(P_{l} f-f\right)-P_{n}\left(P_{l} f-f\right)\right\|_{p}\right)_{n \geq n_{0}}\right\|_{\ell_{q}} \\
& \lesssim\left\|\left(2^{n s}\left\|\left(P_{n} f-f\right)\right\|_{p}\right)_{n \geq l}\right\|_{\ell_{q}} .
\end{aligned}
$$

Therefore, $\lim _{l \rightarrow+\infty}\left\|P_{l} f-f\right\|_{D B_{p, q}^{s}}=0$, due to $f \in D B_{p, q}^{s}$ (Here, $q<\infty$ is needed). Now, it is sufficient to prove

$$
\|f\|_{D B_{p, q}^{s}} \sim\|f\|_{B B_{p, q}^{s}},
$$

to conclude Theorem 4.1.

First, assume $f \in B B_{p, q}^{s}$, then $2^{n s} \omega_{p}^{r}\left(f, 2^{-n}\right) \leq|f|_{B B_{p, q}^{s}}$. By Lemma (3.4), (iii), one knows $\left\|f-P_{n} f\right\|_{p} \leq \omega_{p}^{r}\left(f, 2^{-n}\right)$. Hence $\lim _{n \rightarrow+\infty}\left\|f-P_{n} f\right\|_{p}=0$ for $1 \leq p \leq \infty$. Moreover, $\left\|f-P_{n} f\right\|_{p}=\left\|\sum_{j=n}^{\infty} Q_{j} f\right\|_{p} \leq \sum_{j=n}^{\infty}\left\|Q_{j} f\right\|_{p}$. This with Lemma 4.2 gives

$$
\left\|\left(2^{n s}\left\|f-P_{n} f\right\|_{p}\right)_{n \geq n_{0}}\right\|_{\ell_{q}} \lesssim\left\|\left(2^{n s}\left\|Q_{n} f\right\|_{p}\right)_{n \geq n_{0}}\right\|_{\ell_{q}} .
$$

On the other hand, $\left\|Q_{n} f\right\|_{p} \lesssim 2^{n\left(\frac{1}{2}-\frac{1}{p}\right)}\left\|b_{n}\right\|_{\ell_{p}}$ and $\left\|P_{n_{0}} f\right\|_{p} \lesssim 2^{n_{0}\left(\frac{1}{2}-\frac{1}{p}\right)}\left\|a_{n_{0}}\right\|_{\ell_{p}}$ due to Lemma 3.1, where $Q_{n} f(x)=\sum_{k} b_{n, k} \psi_{n, k}$ and $P_{n} f(x)=\sum_{k} a_{n, k} \varphi_{n, k}$ are defined before Lemma 3.3. Hence,

$$
\left\|\left(2^{n s}\left\|f-P_{n} f\right\|_{p}\right)_{n \geq n_{0}}\right\|_{\ell_{q}} \lesssim\|b\|_{\ell_{p, q}^{s}}
$$

and $\|f\|_{D B_{p, q}^{s}} \lesssim\left(\left\|a_{n_{0}}\right\|_{\ell_{p}}+\|b\|_{\ell_{p, q}^{s}}\right)$. Then $\|f\|_{D B_{p, q}^{s}} \lesssim\|f\|_{B B_{p, q}^{s}}$ follows from Theorem 3.1. 

(4.1).

Next, one needs to show $\|f\|_{B B_{p, q}^{s}} \lesssim\|f\|_{D B_{p, q}^{s}}$ for $f \in D B_{p, q}^{s}$, in order to obtain

Since $\|f\|_{p} \leq\left\|P_{n_{0}} f\right\|_{p}+\left\|f-P_{n_{0}} f\right\|_{p} \leq\|f\|_{D B_{p, q}^{s}}$, one has

$$
\left\|\left(2^{n s} \omega_{p}^{r}\left(f, 2^{-n}\right)\right)_{n \leq n_{0}}\right\|_{\ell_{q}} \lesssim\left\|\left(2^{n s}\|f\|_{p}\right)_{n \leq n_{0}}\right\|_{\ell_{q}} \lesssim\|f\|_{p} \leq\|f\|_{D B_{p, q}^{s}} .
$$

Therefore, it remains to prove

$$
\left\|\left(2^{n s} \omega_{p}^{r}\left(f, 2^{-n}\right)\right)_{n \geq n_{0}}\right\|_{\ell_{q}} \lesssim\|f\|_{D B_{p, q}^{s}} .
$$

Note that $P_{l+1} f-P_{l} f=P_{l+1}\left(f-P_{l} f\right)$ and $f=P_{n_{0}} f+\sum_{l=n_{0}}^{n-1} P_{l+1}\left(f-P_{l} f\right)+(f-$ $\left.P_{n} f\right)$. Then $\omega_{p}^{r}\left(f, 2^{-n}\right) \leq \omega_{p}^{r}\left(P_{n_{0}} f, 2^{-n}\right)+\sum_{l=n_{0}}^{n-1} \omega_{p}^{r}\left(P_{l+1}\left(f-P_{l} f\right), 2^{-n}\right)+\omega_{p}^{r}(f-$ $\left.P_{n} f, 2^{-n}\right)$. Furthermore, it follows that

$$
\begin{gathered}
\omega_{p}^{r}\left(f, 2^{-n}\right) \lesssim 2^{-n\left(r-1+\frac{1}{p}\right)}\left\|P_{n_{0}} f\right\|_{p}+\sum_{l=n_{0}}^{n} 2^{\left(r-1+\frac{1}{p}\right)(l-n)}\left\|f-P_{l} f\right\|_{p} \\
=2^{-n\left(r-1+\frac{1}{p}\right)}\left\|P_{n_{0}} f\right\|_{p}+\sum_{l=-n}^{-n_{0}} 2^{\left(r-1+\frac{1}{p}\right)(-l-n)}\left\|f-P_{-l} f\right\|_{p}
\end{gathered}
$$

from Lemma 4.1 and (ii) in Lemma 3.4. Clearly,

$$
\left\|\left(2^{n s} 2^{-n\left(r-1+\frac{1}{p}\right)}\left\|P_{n_{0}} f\right\|_{p}\right)_{n \geq n_{0}}\right\|_{\ell_{q}} \lesssim\left\|P_{n_{0}} f\right\|_{p}
$$

because of $s<r-1+\frac{1}{p}$. On the other hand, $\|\left(2^{n s}\left[\sum_{l=-n}^{-n_{0}} 2^{\left(r-1+\frac{1}{p}\right)(-l-n)} \| f-\right.\right.$ $\left.\left.P_{l} f \|_{p}\right]\right)_{n \geq n_{0}}\left\|_{\ell_{q}} \lesssim\right\|\left(2^{n s}\left\|f-P_{n} f\right\|_{p}\right)_{n \geq n_{0}} \|_{\ell_{q}}$, due to Lemma 4.2. Hence,

$$
\left\|\left(2^{n s} \omega_{p}^{r}\left(f, 2^{-n}\right)\right)_{n \geq n_{0}}\right\|_{\ell_{q}} \lesssim\left\|P_{n_{0}} f\right\|_{p}+\left\|\left(2^{n s}\left\|f-P_{n} f\right\|_{p}\right)_{n \geq n_{0}}\right\|_{\ell_{q}}:=\|f\|_{D B_{p, q}^{s}} .
$$

Now (4.2) results and the proof is completed.

Remark 4.1. From Theorem 4.1 and $\left\langle\psi_{n, k}, \tilde{\psi}_{n^{\prime}, k^{\prime}}\right\rangle=0$ for $n \neq n^{\prime}$, we know that $\left\langle Q_{n} f, \tilde{\psi}_{n, k}\right\rangle=\left\langle f, \tilde{\psi}_{n, k}\right\rangle$ for $f \in B B_{p, q}^{s}$. Moreover, Theorems 2.1, 3.1 and 4.1 imply Theorem 1.1, which is the main result of this paper.

\section{ACKNOWLEDGEMENT}

The authors would like to thank Professor Gilbert Walter for his many suggestions.

\section{REFERENCES}

[1] K. Bittner, K. Urban, On interpolatory divergence-free wavelets, Math. Comp., 76 (2007), 903-929. MR2291842 (2008b:42067)

[2] A. Cohen, Wavelet Methods in Numerical Analysis, Elsevier, 2003.

[3] D. L. Donoho, Interpolatory wavelet transforms, Preprint, 1992.

[4] R. A. DeVore and G. G. Lorentz, Constructive Approximation, Springer, New York, 1993. MR 1261635 (95f:41001)

[5] T. Hans, Theory of Function Spaces, Birkhauser, Besel, 1983. MR781540 (86j:46026)

[6] W. Härdle, G. Kerkyacharian, D. Picard, A. Tsybakov, Wavelets, Approximation and Statistal Applications, Springer, 1998. MR.1618204 (99f:42065)

[7] R. Q. Jia, Approximation with scaled shift-invariant spaces by means of quasi-projection operators, J. Approx. Theory., 131 (2004), 30-46. MR2103832 (2005h:41035)

[8] R. Q. Jia, Spline wavelets on the interval with homogeneous boundary conditions, Adv. Comput. Math., 30 (2009), 177-200. MR2471447 (2009k:42073) 
[9] R. Q. Jia, J. Z. Wang, D. X. Zhou, Compactly supported wavelets bases for Sobolev spaces, Appl. Comput. Harmon. Anal., 15 (2003), 224-241. MR2010944 (2004h:42042)

[10] Q. Sun, Wiener's lemma for infinite matrices with polynomial off-diagonal decay, C. Acad. Sci. Paris, Ser I, 340 (2005), 567-570. MR2138705 (2005m:42053)

[11] J. Zhao, Wavelet Characterizations for Besov Spaces, Ph. D Dissertation, Beijing University of Technology, P. R. China, 2010.

Department of Applied Mathematics, Beijing University of Technology, Pingle Yuan 100, Beijing 100124, People's Republic of China

E-mail address: liuym@bjut.edu.cn

Department of Mathematics, Tianjin Polytechnic University, 63 Chenglin Street, Hedong District, Tianjin 300160, People's Republic of China

E-mail address: zhaojunjian@emails.bjut.edu.cn 